\title{
The Effects of Seeding Strategies on Morphology of Electroless Deposited Pd Thin Film
}

\author{
T. Gutu ${ }^{1}$, E. Minnaar ${ }^{2}$, J. E. Olivier ${ }^{2}$, J. Kurehwatira ${ }^{1}$, K. Munjeri ${ }^{1}$, B. S. Mpofu ${ }^{1}$ and M. J. Myers $^{1}$ \\ 1. Department of Physics, University of Zimbabwe, Harare, Zimbabwe. \\ 2. Centre for High Resolution Transmission Electron Microscopy, Nelson Mandela Metropolitan \\ University, Port Elizabeth, South Africa.
}

Hydrogen energy carrier has emerged as one of the possible renewable energy candidates to eventually replace the finite fossil fuels [1]. However, hydrogen is produced as a complex mixture of gases called syngas [2]. Hence, separation and purification of hydrogen from syngas is an indispensable process in hydrogen production. Palladium-based membranes have emerged as an attractive technology for separation and purification of hydrogen because of their unique superior properties such as high permeance and theoretically infinite hydrogen selectivity [3]. However, various critical challenges still limit commercial applications of palladium-based membranes. Fabrication of thin dense membranes free of defects such as pinholes or microcracks still remains one of the primary technological challenges. Fabrication of Pd membranes involves the introduction of Pd seeds onto a substrate during the seeding process followed by deposition of a Pd film onto the seeded substrate through a coating technique such as electroless plating. The effects of seeding strategies on the morphology of the electroless deposited Pd film have not been widely investigated. Therefore, the purpose of this investigation is to study the effects of the reducing agent used during the seeding process on the morphology of the final electroless deposited Pd thin film.

A dip coating technique was utilized to introduce $\mathrm{Pd}$ seeds onto glass substrates followed by heat treatment. Various reducing agents were used to reducing palladium acetate to metallic Pd seeds. Hydrazine was used as the reducing agent in the electroless plating that was conducted at $55{ }^{\circ} \mathrm{C}$. An FEI Helios NanoLab DualBeam 650 is a SEM/FIB workstation was utilized to study the morphology of the electroless deposited Pd film at a working distance of 2-5 mm. A JEM-ARM 200F TEM was utilized to analyze the structure and particle size distribution of Pd thin film constituents. To prepare the sample for TEM analysis, a blade was used to scratch the film onto a solution of ethanol, followed by ultrasonication. Then a pipette was used to place a couple of drops of the solution onto the TEM grid.

SEM analysis results showed that Pd thin films formed when hydrogen gas was used as the reducing agent in the seeding step were continuous and the substrate was completely covered (Fig. 1 A \& B). When sodium borohydride reducing agent was used the substrate coverage was not complete as shown in Fig. 1C. The green arrows indicate some of the uncovered areas on the glass substrate which appear as dark areas. A secondary Pd layer grew on top of the first Pd layer. Fig. 1B and D compare the morphologies of the secondary layer nano- to micro-scale particles (shown by the red arrows) of the hydrogen and sodium borohydride derived thin films. The sodium borohydride derived particles were generally much larger and showed flower- and spiky-ball-like morphologies (Fig. 1D) while hydrogen derived secondary layer exhibited chain-like agglomerate morphologies (Fig. 1B). STEM HAADF imaging showed the presence of large ( 400-500 nm) Pd agglomerates (Fig. 2A) and Pd nanoparticles (Fig. 2B) in the film irrespective of the reducing agent used. Most of the nanoparticles had a diameter of between $0.5 \mathrm{~nm}$ to $3 \mathrm{~nm}$. In summary, this investigation showed that the reducing agent used during the seeding step determine the morphology and substrate coverage of the Pd thin film. 


\section{References:}

[1] K Bennaceur et al, Oilfield Review (2005), p. 30-41.

[2] J Turner et al, Energy Research 32 (5) (2008), p. 379-407.

[3] E Kikuchi, Catalysis Today (2000), 56 (1-3), p. 97-101.

[4] Authors acknowledge funding from the University of Zimbabwe Research Board grant No: RB36 and The World Academy of Sciences research grant agreement No: 14-108 RG/PHYS/AF/AC_I-

UNESCO FR: 324028571. The electron microscopy analysis was done at Nelson Mandela Metropolitan University's Centre for High Resolution Transmission Electron Microscope
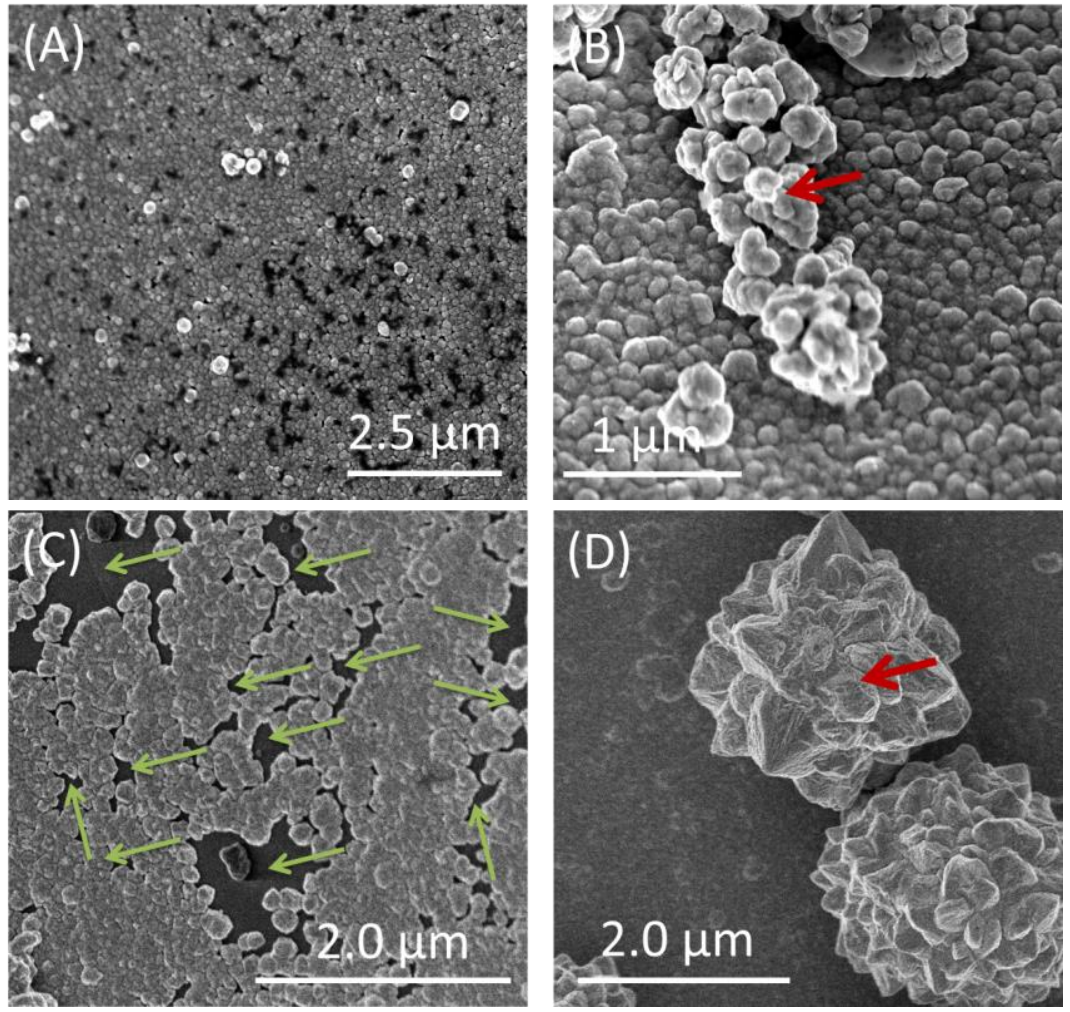

Figure 1. SEM images of $\mathrm{Pd}$ thin films prepared with different reducing agents during the seeding stage: A-B hydrogen gas reducing agent and C-D sodium borohydride reducing agent.
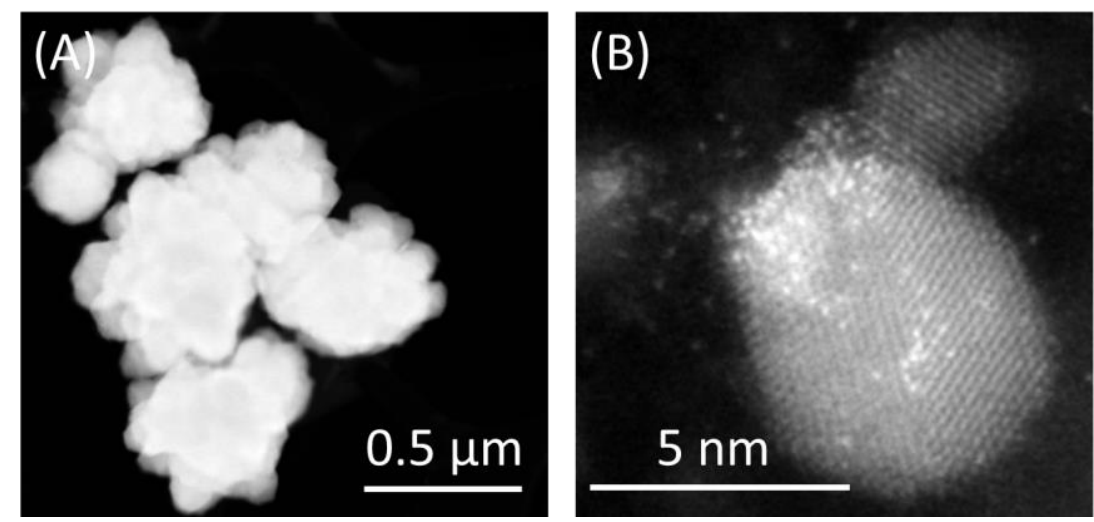

Figure 2. STEM HAADF images: (A) Pd agglomerates and (B) Pd nanoparticles. 